\title{
Correlation Between Monitoring Practices During Anesthesia and Patient Recovery
}

\author{
Tariq No'aman Al-Shurbaji ${ }^{{ }^{*}}$, Mohammed Al-Falaki ${ }^{2}$
}

${ }^{1}$ Senior Specialist Anesthesiologist and Training Coordinator of Residency Program in Anesthesia and Intensive Care, Dept of Anaesthesia,Salmaniya Medical Complex, Kingdom of Bahrain

${ }^{2}$ Professor of Anaesthesia and Senior Consultant, Dept of Anaesthesia, Salmaniya Medical Complex, Kingdom of Bahrain

Correspondence to: Tariq No'aman Al-Shurbaji, Senior Specialist Anesthesiologist and Training Coordinator of Residency Program in Anesthesia and Intensive Care, Dept of Anaesthesia,Salmaniya Medical Complex, Kingdom of Bahrain.

Received date: Januyary 17, 2021; Accepted date: Januyary 30, 2021; Published date: February 6, 2021

Citation: Al-Shurbaji TN, Al-Falaki M (2021) Correlation Between Monitoring Practices During Anesthesia and Patient Recovery. J Clin Anesthes Res 2(1): pp. 1-4. Copyright: (C2021 Al-Shurbaji TN, et al. This is an open-access article distributed under the terms of the Creative Commons Attribution License, which permits unrestricted use, distribution and reproduction in any medium, provided the original author and source are credited.

\section{ABSTRACT}

Anesthesia is used by healthcare professionals to induce loss of sensation in the body of the patient so that the surgical process of treatment is carried out effectively. Anesthesia doses that are given to patients have been classified into three types which are general anesthesia, sedation anesthesia, and regional or local anesthesia. These medications are given to patients as per the severity of the injury and treatment process. The provision of anesthesia includes monitoring processes such as Electrocardiography (ECG), Noninvasive Blood Pressure (NIBP), pulse oximetry, and others so that patient safety is ensured at all the levels of treatment. It also includes Depth of General Anesthesia (DGA) is an advanced monitoring process of anesthesia through which accurate drug administration in the individual patient is executed. The study introduces current prospects of anesthesia monitoring and mentions details about important methods and technologies in use and their both advantages and disadvantages. It also provides details about DGA and other latest upcoming methods for anesthesia monitoring.

\section{Keywords:}

Anesthesia, Monitoring, Depth of General Anesthesia (DGA), Electrocardiography (ECG), Oscillometric method

\section{Abbreviations:}

ECG: Electrocardiography; DGA: Depth of General Anesthesia; NIBP: Noninvasive Blood Pressure; BP: Blood Pressure; AEP: Acoustic Evoked Potentials; CVP: Central Venous Pressure

\section{Introduction}

Anesthesia is defined as the condition in which the dosed individual experiences a temporary loss of sensation because of the drug effect. It has been classified into three types which are general anesthesia, sedation anesthesia, and regional or local anesthesia which must be given to the patient under the strict guidance of healthcare experts. While focusing on the current status of monitoring methods that are in use for anesthesia regulation, it also includes several standards that are set by the American Society of Anesthesiologist (ASA) to monitor the anesthetic patients. The first standard includes the presence of qualified anesthesia personnel in the ward where the patient is treated by using anesthesia so that throughout monitoring of the patient is executed. The second standard includes the use of certain processes such as oxygenation, ventilation, circulation, and others to observe the changes experienced by the patient before, anesthesia, and after anesthesia dose.

In order to make focus on the basic aspects based on Anesthesia; it includes three basic aspects which are oxygenation that mainly supervises the circulatory and respiratory functioning. Ventilation assessment is used to monitor respiratory functioning and the Circulation assessment procedure. It is used to monitor respiratory functioning related to cardiac output. Moreover, as per the ASA standards, the monitoring of the anesthetic patients is a continuous process that is carried out by using several procedures such as pulse oximeter, electrocardiography, noninvasive blood pressure device, and a temperature monitor. The other process such as Electrocardiography (ECG), Oscillometric method, Respirometry assessment, Blood Pressure (BP) monitoring that is also included in the monitoring of practices during anesthesia and patient recovery. For example, ECG monitoring assesses the alterations that occur in heart rate. On the other hand, a pulse oximeter is used to measure the levels of oxygen saturation in the blood. However, there are limitations such as changes in pulse oximeter readings when assessing patients who are suffering from lung disease or asthma or smoking patients. In such specific cases, intensive and specific intervention is required to evaluate the blood and oxygen saturation so that heartbeats are displayed properly. Moreover, there are so many difficulties such as slowing of breath or stopping of breath when the patient is under the effect of anesthesia that causes hindrances in the effective monitoring practices during anesthesia dose. It impacts the working of the anesthesiologist as they have to use other measures such as ventilation and oxygenation to assess the condition of patients. Hence due to limitations such as inappropriate monitoring practices like the absence of supervision by experts during anesthesia, one individual in every 1,00,000 general anesthetic patients dies.

Depth Of General Anesthesia (DGA) is an advanced monitoring process of anesthesiathrough which accuratedrugadministration in the individual patient is executed. The DGA monitoring helps in enhancing monitoring practices during anesthesia and patient recovery and provides improved patient recovery outcomes by preventing awareness or excessive anesthetic depth. The DGA monitoring involves clinical monitoring that takes clinical 
signs, skin conductance, isolated forearm technique, heart rate variability, Ramsey scale, and Modified Observer Assessment of Alertness/Sedation scale (MOAA/S) into account. DGA is a continuous monitoring evaluation process performed by using electroencephalogram (EEG ), Acoustic Evoked Potentials (AEP) and based monitor systems. The application of DGA monitoring helps in interpreting the results that are procured by EEG or AEP evaluations. Moreover, new approaches such as advanced supraglottic airway devices, airway algorithms, video laryngoscopy, and others have been commenced to monitoring practices during anesthesia and patient recovery. Hence, it can be said that the current study examines the correlation that exists between monitoring practices during anesthesia and patient recovery and investigates anesthesia monitoring processes along with limitations of the current monitoring of anesthesia methods.

\section{Aim and Objectives}

The objectives of the study to achieve the ultimate aim of the study are:

- To introduce current prospects of anaesthesia monitoring

- To mention details about important methods and technologies in use and their both pros and cons.

- To introduce details about DGA and other latest upcoming methods for anesthesia monitoring.

\section{Literature Review}

\section{Current anesthesia monitoring strategies}

The monitoring in anesthesia has become a subject of research and development as it is directly associated with good health conditions of patients. Therefore, it is recommended by global organizations such as the Association of Anesthetists of Great Britain and Ireland (AAGBI), American Society of Anesthesiologists (ASA) to implement anesthesia monitoring techniques in the perioperative, during and post-operative period so that safe treatment is provided to patients. There are several processes of anesthesia monitoring such as Electrocardiography (ECG), blood pressure monitor, pulse oximeter, breathing monitors, anesthetic monitors, brain activity monitor, and others. Electrocardiography (ECG) evaluates and records the modifications that occur in heart rate and rhythm.

It also estimates the risk factors associated with the thickening of the heart muscles because of high blood pressure. ECG monitoring is also used to investigate the presence of blockages in the coronary arteries and ascertains the risk of a heart attack under serious complications. While blood pressure monitor equipment is used to examine the pressure in the blood vessels when the heart contracts. It uses both invasive and non-invasive techniques to examine the pressure of blood in the heart when the patient is under the influence of anesthesia dose. The invasive blood pressure is measured by placing the catheter inside an artery and not carried out in the case of routine surgery. On the other hand, non-invasive blood pressure monitoring is carried out by using a cuff that is pressed around the arm. It helps in estimating blood pressure and detects the changes that are experienced by the patient when sleeping.

Pulse oximeter also named as a non-invasive technique is used for estimating oxygen saturation levels in the blood among the patients at the time or during anesthesia. The non-invasive techniques such as Oscillometric blood pressure measurement are also used to indirectly measure the blood pressure by using an electronic display and control unit that is connected to the appropriate sized cuff. The non-invasive technique includes a Doppler ultrasonic detection method in which blood flow and pressure are measured by using ultrasonic crystal and transducer. The device is placed over blood vessels such as a palmar metacarpal artery so that the changes that are occurring in the blood flow and pressure are recorded. While the breathing monitors are assessed with the help of a ventilator through which rate, depth, and pattern of respiration are monitored. The ventilator also monitors and displays the percentage of gas that is sent to the patent so that there is right delivery of gas volume and the lungs are not over or under expanded when breathing of the patient is assisted by using a ventilator. In addition to this, anesthetic monitoring is carried out to measure the volume of anesthesia gas and displays the correct readings on anesthesiologists' monitor. On the other hand, Brian Activity Monitor (BIS) is used to indicate the depth of anesthesia and known as a modified version of EEG. It also provides relevant and limited facts about the supply of oxygen to the brain.

Anesthesia monitoring procedures such as continuous invasive arterial blood pressure, Central Venous Pressure (CVP), TOE, NMM, and others are also used to monitor the patient recovery during anesthesia dose. While implementing the different anesthesia monitoring processes, pulse oximetry has been identified to be most suitable and used by the majority of clinicians around the world. The pulse oximetry anesthesia monitoring process has been tried upon more than 21,000 patients that have helped in the successful identification of hypoxemia and other related facts about the patients.

\section{Advantages and disadvantages of anesthesia monitoring strategies}

Anesthesia is highly used for medical purposes under the influence of which the patient loses its sensation and may experience paralysis, amnesia, or unconsciousness when not monitored properly. Therefore, it is essential to implement patient monitoring so that patient safety is ensured at each level of treatment. In anesthesia monitoring practices at the time of patient recovery, there must be accurate maintenance of records related to oxygen saturation, heart rate, blood pressure, anesthetic vapor concentration, and other aspects as they are vital for patient safety.

The use of handover checklist along with several anesthesia monitoring practices such as Non-Invasive Intermittent Blood Pressure (NIBP), ECG, pulse oximeter, and others helps inappropriate monitoring of the patients. It also includes the guidelines that are given by the Association of Anesthetists of Great Britain and Ireland (AAGBI) and the American Society of Anesthesiologists (ASA) regarding the anesthesia monitoring process and ensuring patient safety. The ASA guidelines encourage the use of continual monitoring process so that there is the effective use of anesthesia monitoring practices in the form of automatic noninvasive blood pressure measurement, ECG monitoring so that there is uninterrupted monitoring of the anesthesia patients.

Due to the implementation of adequate monitoring practices during anesthesia and patient recovery, there has been a 
reduction in the anesthetic mortality rates over 25 years. The reports indicated 1 death against 2,680 patients in the year 1959 which has reduced to 1 death against every 10,000 anesthesia patients in the year 1980. . It was also found that due to proper adoption of monitoring practices during anesthesia and patient recovery, cardiac arrest cases have also reduced from 15 deaths against 20,080 patients to the death against $2500-3000$ patients who were under the dose of anesthesia. In addition to this, the risk associated with the percentage of per operative death with respect to the usage of general anesthesia has reduced from $33 \%$ to $1 \%$. Hence, it can be said that the use of monitoring practices during anesthesia and patient recovery has helped in saving the lives of the patients and reduce mortality rates because of inappropriate monitoring practices during anesthesia to a minimum.

The use of general anesthesia in the clinical process may cause certain ailments such as vomiting, nausea, headache and late recovery of the consciousness which may impact the good health conditions of the patients. In addition to this, anesthesiologists also find it difficult to measure and record the several physiological fluctuations that are experienced by the patient under the influence of general anesthesia.

It may also occur that under general anesthesia, the patient suffers from malignant hyperthermia that increases the temperature of the body with the response with an anesthetic medication. There are issues with the implementation of monitoring practices during anesthesia and patient recovery as they do not provide a correct assessment of the condition of a patient under the influence of anesthesia. For example, anesthesia monitoring method such as BIS has several limitations such as non-reflection of individual patient requirement and inaccurate estimation of large dose opioids. It also includes an inappropriate reflection of the implications of supplementary regional anesthesia and improper estimation of blood pressure. As a result, there is unnecessary monitoring end-tidal concentration and inaccurate reflection of the level of anesthetic dose among the patients. It creates issues in the rightful assessment of the patient's condition and therefore, essential to introduce fitting monitoring practices during anesthesia and patient recovery so that patient outcomes could be evaluated rightfully.

\section{DGA and its importance}

Anesthesia is highly used for medical purposes under the influence of which the patient loses its sensation and may experience paralysis, amnesia, or unconsciousness when not monitored properly. Therefore, it is essential to implement patient monitoring so that patient safety is ensured at each level of treatment. In anesthesia monitoring practices at the time of patient recovery, there must be accurate maintenance of records related to oxygen saturation, heart rate, blood pressure, anesthetic vapor concentration, and other aspects as they are vital for patient safety.

The use of handover checklist along with several anesthesia monitoring practices such as non-invasive intermittent blood pressure (NIBP), ECG, pulse oximeter, and others helps inappropriate monitoring of the patients. It also includes the guidelines that are given by the Association of Anesthetists of Great Britain and Ireland (AAGBI) and the American Society of
Anesthesiologists (ASA) regarding the anesthesia monitoring process and ensuring patient safety. The ASA guidelines encourage the use of continual monitoring process so that there is the effective use of anesthesia monitoring practices in the form of automatic noninvasive blood pressure measurement, ECG monitoring so that there is uninterrupted monitoring of the anesthesia patients.

Due to the implementation of adequate monitoring practices during anesthesia and patient recovery, there has been a reduction in the anesthetic mortality rates over 25 years. The reports indicated 1 death against 2680 patients in the year 1959 which has reduced to 1 death against every 10,000 anesthesia patients in the year 1980. It was also found that due to proper adoption of monitoring practices during anesthesia and patient recovery, cardiac arrest cases have also reduced from 15 deaths against 20080 patients to the death against $2500-3000$ patients who were under the dose of anesthesia. In addition to this, the risk associated with the percentage of per operative death with respect to the usage of general anesthesia has reduced from $33 \%$ to $1 \%$. Hence, it can be said that the use of monitoring practices during anesthesia and patient recovery has helped in saving the lives of the patients and reduce mortality rates because of inappropriate monitoring practices during anesthesia to a minimum.

The use of general anesthesia in the clinical process may cause certain ailments such as vomiting, nausea, headache and late recovery of the consciousness which may impact the good health conditions of the patients. In addition to this, anesthesiologists also find it difficult to measure and record the several physiological fluctuations that are experienced by the patient under the influence of general anesthesia. It may also occur that under general anesthesia, the patient suffers from malignant hyperthermia that increases the temperature of the body with the response with an anesthetic medication. There are issues with the implementation of monitoring practices during anesthesia and patient recovery as they do not provide a correct assessment of the condition of a patient under the influence of anesthesia. For example, anesthesia monitoring method such as BIS has several limitations such as non-reflection of individual patient requirement and inaccurate estimation of large dose opioids. It also includes an inappropriate reflection of the implications of supplementary regional anesthesia and improper estimation of blood pressure. As a result, there is unnecessary monitoring end-tidal concentration and inaccurate reflection of the level of anesthetic dose among the patients. It creates issues in the rightful assessment of the patient's condition and therefore, essential to introduce fitting monitoring practices during anesthesia and patient recovery so that patient outcomes could be evaluated rightfully.

\section{Standard and upcoming techniques in the clinical setting for anesthesia monitoring}

The standard anesthetic monitoring process includes elements such as oxygenation, ventilation, circulation, the temperature so that there is an effective measurement of the anesthesia dose implications. While focusing on the circulation process, it is monitored by using anesthesia monitoring practices such as Electrocardiography (ECG), Ultrasonic Doppler blood flow detector, Oscillometric BP measurement, and Invasive BP 
measurement during anesthesia and patient recovery. These processes help in the monitoring of heart rate, diagnosing arrhythmias, estimating systolic and assessing other health parameters of the patients. The ventilation process includes the use of anesthesia monitoring practices such as Respirometer, Arterial or venous blood gas, or Capnography that helps in measuring respiratory rate, the partial pressure of carbon dioxide, end-tidal carbon dioxide concentration, and others. The oxygenation process includes the use of anesthesia monitoring practices such as Pulse oximetry and Arterial blood gas that helps in estimating saturation of oxygen bound to hemoglobin (spo2) and partial pressure of oxygen ( $\mathrm{PaO} 2$ ) in arterial blood samples of the patients who are under the influence of anesthesia. The body temperature is measured by using a rectal thermometer, esophageal temperature probe, and Infrared thermometer so that tympanic membrane temperature is estimated accurately. In addition to this, depth of anesthesia is also evaluated by using anesthesia monitoring practices such as an Anaesthetic gas analyzer and Bispectral index (BIS) monitor.

It helps in the measurement of expiratory inhalant concentration, expression of algorithmic, and analysis of electroencephalogram when general anesthesia dose is given to the patient. Hence, it can be said that the use of these standards helps in the enhanced implementation of monitoring practices during anesthesia and patient recovery. The significant improvements have been brought in the monitoring processes of anesthesia so that the care of the patients could be taken properly. It includes the patients who are undergoing surgery also those who are kept in the Intensive Care Units (ICUs). The introduction of new monitoring practices during anesthesia and patient recoveries such as extubation catheter, advanced supraglottic airway devices, airway algorithms, and others also helps to enhance the monitoring process of anesthesia and arresting the mortality rates. For example, the new monitoring practices during anesthesia and patient recovery such as perioperative cardiac monitoring help in providing more safety to the patient treatment process by acquiring readings related to the valvular function, hemodynamic parameters, and others by using 3D-echocardiography. Hence, it can be said that advances in the monitoring process of anesthesia help in improving the safety condition of the patients.

\section{Conclusion and Recommendations}

As per the above-discussed literature, it was found that the rightful monitoring of patients during anesthesia is essential for their recovery and estimation of the exact health condition of patients. The study also examined that the rightful assessment of blood pressure, heartbeat rate, body temperature is necessary to ensure patient safety. It was also examined that monitoring practices during anesthesia and patient recovery are carried out by using several monitoring procedures such as ECG, pulse oximetry, a nerve stimulator, and others so that patient safety is ensured at each stage of treatment and surgery.

The study also examined that the depth of general anesthesia is necessary to be included so that there is an accurate estimation of the effect of anesthesia on the central nervous system. Moreover, the implementation of monitoring practices during anesthesia and patient recovery reduces the risks associated with surgery and safeguards the good health conditions of the patients undergoing surgery. Additionally, the Association of Anesthetists of Great Britain and Ireland (AAGBI) suggested that the standards that are provided by the organization are necessary to be followed so that the efficacy of the monitoring practices during anesthesia and patient recovery increases. The organization suggests that proper care of the patients who are under the influence of anesthesia must be taken by recording their heartbeat, blood flow levels, blood pressure, and other health parameters. It is also recommended that alarm limits must be set before the use of the monitoring equipment by carrying out pre-hand checking so that the efficacy of the devices improves. In addition to this, there must be involvement of expert anesthetist for the provision of anesthesia to the patient and monitoring during the anesthesia and patient recovery.

\section{References}

1. Yeoh TY, Manninen P, Kalia SK, et al. (2017) Anesthesia considerations for patients with an implanted deep brain stimulator undergoing surgery: a review and update. Can J Anesth 64(3): pp. 308-319.

2. Doshi PK (2018) Expanding indications for deep brain stimulation. Neurol India 66(Suppl): pp. 102-112.

3. Gandhi R, Chawla R (2014) Anaesthetic management of shoulder arthroscopic repair in Parkinson's disease with deep brain stimulator. Indian J Anaesth 58(3): pp. 309-311.

4. Khetarpal M, Yadav M, Kulkarni D, et al. (2014) Anaesthetic management of a patient with deep brain stimulation implant for radical nephrectomy. Indian J Anaesth 58(4): pp. 461-463.

5. Singh M, Venkatraghavan L (2018) Cortical arousal with deep brain stimulation after general anesthesia for laparoscopic cholecystectomy. J Neurosurg Anesthesiol 30(1): pp. 77-78. 